\title{
Experiences Receiving HIV-Positive Results by Phone: Acceptability and Implications for Clinical and Behavioral Research
}

\author{
Alexa B. D'Angelo ${ }^{1,2}$. Corey A. Morrison ${ }^{2}$. Javier Lopez-Rios ${ }^{1} \cdot$ Caitlin J. MacCrate $^{3} \cdot$ David W. Pantalone $^{4,5}$. \\ Matthew Stief ${ }^{2} \cdot$ Christian Grov $^{1,2}$
}

Published online: 11 September 2020

๑) Springer Science+Business Media, LLC, part of Springer Nature 2020

\begin{abstract}
Improving HIV testing rates and increasing early detection among men who have sex with men (MSM) are critical strategies for enhancing overall health and decreasing HIV transmission. Remote testing and phone delivery of HIV test results may reduce barriers such as geographic isolation or HIV-related stigma. In 2018-19, 50 MSM completed qualitative interviews about their experience receiving a positive HIV test result via phone through their participation in a research study that included remote HIV testing. Interview topics included the acceptability of, and concerns about, phone delivery of HIV results, as well as suggestions for improvement. Interviews were transcribed, coded, and analysed using an inductive thematic approach. Overall, participants reported high acceptability of phone delivery of HIV-positive results. Participants praised the support and information provided by study staff. Benefits identified included increased convenience compared to in-person medical visits, allowing participants to emotionally process their test results privately, as well as receiving the results from supportive and responsive staff members. A few participants indicated drawbacks to phone-based HIV test result delivery, such as logistical concerns about receiving a phone call during the day (e.g., while at work), reduced confidentiality, and the lack of in-person emotional support. Overall, participants described phone delivery of positive HIV-results as acceptable. At-home testing with phone delivery has the potential to increase HIV testing access, especially to geographically isolated or medically underserved patients.
\end{abstract}

Keywords HIV · MSM · HIV testing · Qualitative research · Telemedicine

\section{Introduction}

As the HIV epidemic enters its fourth decade, men who have sex with men (MSM) continue to be overrepresented among new HIV diagnoses. The U.S. Centers for Disease Control and Prevention (CDC) reported that adult and adolescent

Christian Grov

cgrov@sph.cuny.edu

1 CUNY Graduate School of Public Health and Health Policy, 55 W. 125th St., 7th Floor Mailroom, New York, NY 10027, USA

2 CUNY Institute for Implementation Science in Population Health, New York, NY, USA

3 Graduate School of Education, Fordham University, New York, NY, USA

4 University of Massachusetts Boston, Boston, MA, USA

5 The Fenway Institute, Fenway Health, Boston, MA, USA
MSM represented nearly $70 \%$ of new HIV diagnoses in 2017 , despite only constituting approximately $3.6 \%$ of the general population [1,2]. As of 2006, the CDC formally recommended that sexually active MSM be screened for HIV at least annually, with more recent clinical guidelines suggesting even more frequent testing [3]. However, recent studies and national survey estimates approximate that $8-17 \%$ of MSM have never tested for HIV [1, 4-7]. Additionally, recent data indicate that nearly one-in-six MSM living with HIV are currently unaware of their status [1]. Thus, utilization of HIV screening services are suboptimal for MSM, contributing to new infections and poorer health outcomes for MSM living with HIV [8-12].

Pertinent barriers to optimal HIV testing include concerns about the confidentiality of HIV testing in a healthcare facility [13], prevalence of HIV-related stigma [14], and fear of testing positive [15]. Further, healthcare access issues, including a lack of insurance or underinsurance $[16,17]$, inadequate access to culturally competent 
primary care and/or testing facilities [18, 19], and inadequate transportation act as structural barriers to HIV testing, particularly for low-income MSM across the U.S [18]. Additionally, geographic isolation or rurality is associated with significantly lower rates of HIV testing among MSM compared to urban populations [20]. Some of these barriers also contribute to patients not returning to receive HIV test results after the test is performed. That said, although the ubiquity of rapid, clinic-based HIV testing overcomes the need to return for HIV test results for many, it does not obviate barriers related to geographic isolation, healthcare access, and stigma, which remain barriers to clinic-based testing [14, 19, 21-23]. Given the importance of increased testing and early HIV diagnosis, at-home HIV testing and results delivery could be a helpful strategy for increasing HIV testing for MSM.

Technological advances in at-home test kits have made remote HIV testing and results delivery more feasible. FDAapproved, rapid at-home HIV test kits allow individuals to complete an HIV test from home by collecting an oral fluid or blood sample and receiving preliminary results within minutes [24]. Further, oral fluid and blood sample collection devices allow individuals to collect their sample at home, mail it to a lab, and access their test results several days later via web-based portal [24, 25]. Rapid at-home testing and mail-in testing may help overcome some of the logistical challenges of in-person testing, including forgoing the need to travel to a clinic, and alleviating systemic barriers to healthcare access, like lacking a provider, clinic, or health insurance. However, at $\$ 40-\$ 80$, the cost of rapid and mailin home testing remains prohibitive for many, especially for MSM who are recommended to test every 6 months or more frequently $[25,26]$.

Rapid at-home testing may not be acceptable for all MSM. Some men may be concerned about receiving a positive test result while they are alone, and may require interpersonal contact and support to process an HIV-positive result. One study found that, although rapid home testing was acceptable to most MSM in their sample, $23 \%$ of participants were concerned about receiving their results alone [27]. Mail-in services like myLAB use a web-based portal to deliver HIV-positive test results, with the option of requesting free HIV counseling [25]. However, this structure places the onus on individuals to reach out for counseling and referral-tasks that could be difficult for those who are emotionally distressed due to an HIV-positive result. Further, results from another study found that most participants believed a 24-h hotline for counseling was not enough support for those testing HIV-postive [28]. Thus, phone delivery of HIV-positive results may help to remove some obstacles to testing while maintaining the social support and immediate referral to care that are core elements of in-person HIV counseling.
Further study of the delivery of HIV positive results via phone is warranted due to the availability of mail-in HIV test kits, as well as its potential to alleviate some of the key barriers to clinic-based testing, especially the need to return to receive HIV test results. Although phone delivery of HIVpositive results has been available for the better part of a decade, there remains a paucity of data on the subject. In part, the dearth of literature is a result of there being (as far as we could identify) no official guidelines from the CDC or other governing institutions for the delivery of HIV positive results by phone. Apprehension and norms among public health and HIV experts from earlier in the HIV epidemic have hindered progress in this area due to concerns about suicide risk among those receiving results remotely [29]. However, these concerns have not been substantiated by data [30].

Additionally, the increased emergence of fully-online HIV prevention studies, and greater emphasis on biospecimen testing over self-report, further necessitates increasing knowledge about the acceptability and feasibility of delivering HIV-positive results via phone [31]. In 2017, the National Institutes of Health funded multiple research sites to conduct Limited Interaction Targeted Epidemiology (LITE) to Advance HIV Prevention [32]. Funded sites are each following thousands of participants through 2022, and at-home HIV testing is an integral component of study participation [32-34]. HIV prevention studies operating remotely have the option of using rapid or mail-in HIV testing. However, mail-in testing overcomes some logistical challenges. For example, chances of non-reporting of HIV results may be higher among individuals tasked with reporting results, versus a contracted lab delivering results directly to study coordinators. Further, phone-delivery of HIV-positive results maintains a higher-level of engagement with participants, making immediate connection to care more feasible. Those benefits make it preferred by some researchers and public health programmers.

Prior research on the acceptability and feasibility of receiving preliminary HIV test results by phone is limited, and research specifically focused on giving or receiving HIV-positive test results is nearly absent. In a 2002 study of vulnerable, homeless youth in Portland, Oregon, participants were randomized either to receive HIV test results over the phone or in a traditional medical setting. Overall, participants reported barriers to receiving HIV results in traditional medical settings, and were 2.3 times more likely to receive test results if given the option of phone notification [35]. Of note, this study excluded participants who tested HIVpositive. Similarly, McKinstry et al. found that participants offered phone results delivery were 2.5 times more likely to receive HIV results than those not offered the option. For participants who tested HIV positive, rates of receiving results increased from $85 \%$ to $94 \%$ [36]. A study conducted 
at an Australian health clinic found a 97\% satisfaction rate among participants who elected to receive their HIV result over the phone [37]. Although these studies provide preliminary evidence of general acceptability of receiving HIV test results over the phone, only McKinstry et al. included participants who received a positive result by phone, leaving significant gaps in the current literature [36].

Overall, there is considerable need to evaluate the acceptability of phone delivery of HIV-positive results within key populations, especially those that appear to be both at high risk of HIV infection with a relatively low rate of testing, such as MSM. In this study, we aim to assess the experiences of MSM who received a preliminary positive HIV test result over the phone in the context of a remote HIV prevention study. The data used for this study are primarily qualitative, allowing us to explore participants' experiences in their own words.

\section{Methods}

\section{Participants and Procedures}

Data are taken from Together 5000 (herein T5K), a U.S. national, internet-based cohort study of cisgender men, trans men, and trans women who have sex with men. The goal of T5K is to identify modifiable individual and structural factors associated with HIV seroconversion. Enrollment began in October 2017 using ads on men-for-men geosocial networking phone applications (apps) and concluded in June 2018. The cohort and study procedures have been fully described elsewhere [33, 38, 39]. Briefly, core eligibility criteria for enrollment specified that participants were aged 16 to 49 ; had at least two male sex partners in the prior 3 months; were not currently participating in an HIV vaccine or PrEP clinical trial; were not currently taking PrEP; lived in the U.S. or its territories; were not known to be HIVpositive; had a gender identity other than cisgender female; and reported behavioral risk for HIV.

Of those who completed the enrollment survey, 8,755 participants met eligibility criteria. These participants were invited to complete a baseline online survey via email. Of those, 6267 (71.6\%) completed the baseline survey and received a $\$ 15$ incentive $[38,39]$. Following completion of the survey, and for an additional \$15 incentive, participants were mailed an at-home biospecimen collection device. We used the OraSure HIV-1 specimen collection device. Collection procedures involved taking an oral swab and placing it in an oral fluid container, and mailing the specimen, using provided prepaid shipping materials, to the New York State Department of Health (Avioq HIV-1 Microelisa System). At baseline, we successfully delivered 6150 HIV test kits to participants, 5065 of which were returned by the lab. In total, 195 participants had a reactive HIV-positive test result at enrollment. Participants were not told in advance specifically how they would be contacted to receive their results. HIV-negative results were sent to participants via email. Preliminary HIV-positive results were delivered to participants via phone. HIV-positive results delivery followed a studycreated protocol that included provision of referrals to local healthcare resources to facilitate confirmatory testing and linkage to care (See Table 1 for Protocol).

The T5K HIV-Positive Results Delivery and Emergency Protocol was developed by CJM, a school psychology $\mathrm{PhD}$ candidate, with the supervision of DWP, a clinical psychologist with expertise in HIV counseling. CJM's clinical training from The Fordham University Graduate School of Education was used to inform the development of our clinical protocol, as well as her New York State training in HIV results delivery. Additionally, our protocol was informed by The Fenway Institute's Standard Operating Procedure for HIV Counseling and Testing, as well as the CDC Revised Guidelines for HIV Counseling, Testing, and Referral [29]. Our emergency procedures were adapted from the Columbia-Suicide Severity Rating Scale (C-SSRS) [40]. The C-SSRS assesses risk of suicide and is used in our protocol to assess participant risk during phone calls through a series of questions. If participants revealed intent to harm themselves or others, staff followed the emergency procedures, which included wellness checks from emergency services located near participants.

Prior to each phone delivery, trained staff curated local testing facilities' information, as well as emergency services, which were on hand, if required. Testing facilities were vetted by staff members via phone, and a list of questions were asked of each facility prior to referring participants for testing. Clinic screening questions include an assessment of free or low-cost confirmatory testing, as well as appointment availability for new or uninsured patients (see Table 1 for list of items asked). Emergency wellness check contact information was also curated prior to each results delivery, and included resources available near the individual participant. The T5K HIV-Positive Results Delivery and Emergency Protocol was developed with consideration of our participants' unique needs and available resources, with the recognition that resources vary greatly by state and region. Additionally, the protocol aimed to meet participants where they were at, by providing HIV-positive test results with sensitivity and cultural-competency. The protocol was also developed with a social scientific understanding of the intersecting vulnerabilities faced by many in our study population.

Staff provided information about local HIV testing resources, a referral to HIV care, and basic information about HIV treatment. Of note, delivery conversations always included a description of HIV as a manageable disease with 
Table 1 The T5K HIV-Positive Results Delivery and Emergency Protocol

Things to have on hand when making an HIV + results delivery call

- Local emergency services: welfare, behavioral response team, medical

- Local testing services: details including how to make an appointment, office hours, LGBT competence, transit accessibility, name and number of point-person to call, what insurance they take, whether they accept patients without insurance, mental health referral services

Leaving a Voicemail

1. Voicemail: This is me a call back at

calling from the CUNY School of Public Health, calling about your participation in a research study. Please give . That's . Thanks! Bye.

Sending a Text

1. Text participant: introduce self as part of Together 5000. Schedule convenient time to talk.

Phone Call Script

1. Confirm who you are speaking with

a. Hi, this is [Staff Member] from the The CUNY School of Public Health, Is [NAME] available?

2. Deliver the results

a. Ok, great. This is [Name] from Together 5,000, the research study you joined. How are you doing today?

b. Is now a good time to talk?

c. Where are you? At home? Would it be possible for you to move to a private location? I want to make sure that we can hear each other well, and that you can ask whatever questions you might have without worrying about being overheard.

d. Do you remember Together 5,000?

e. So you remember that HIV testing was part of the study. The results came back and suggest that you DO have the virus.

f. [Pause]

3. Establish rapport, assess participant reaction

a. How are you feeling right now?

b. What did you think the results were going to be? (To get them talking, so that the staff member can respond to misinformation, provide guidance about next steps, etc.)

c. Do you know anyone living with HIV? (Get them to start imagining healthy living with HIV, connection to potential social supports)

d. What do you know about HIV treatment/medication? (To provide the staff person the opportunity to educate the participant about the current state of living with HIV, i.e., as a livable chronic condition, like diabetes.)

4. Connect to confirmatory testing and treatment

a. The test result I've given you today is called a "preliminary positive" from a screening test. That means that you will need confirmatory testing-which is a second test that you take to confirm your test results.

i. [If they ask for details] This test tests for the presence of HIV antibodies. Antibodies are the cells that a body produces in response to HIV. These antibodies can take up to 3 months to reach a level that the test can detect, so the test is only testing exposure to HIV you had from 3 months ago and earlier. This test is very accurate, which is good, but you still need to go for confirmatory testing. Basically, the confirmatory test is an even more accurate test for HIV and can give you clearer information about your status.

b. HIV is completely treatable, and HIV + people live completely healthy and normal lives with treatment. It's important to get the results confirmed and get connected to treatment as soon as possible. There are lots of resources out there that make treatment either free or affordable.

c. Do you have health insurance?

d. Do you have a primary care doctor who you see regularly?

i. [If yes] Would you be comfortable going for a visit with this doctor to have your confirmatory test?

1. Ok, great. I can also send over information about other places in case you want to get the confirmatory test somewhere else.

ii. [If no] I know of a clinic nearby, . Have you heard of it? There is a person who works there, [Name], who can meet you to do this. They are super nice over there. I just got off the phone with them.

1. Do you want me to email this info, text it to you, or do you want to write it down now while we are talking?

e. Do you have any questions for me?

f. What does the rest of your day look like?

i. Would you want to maybe invite someone over? Who would you think about having over? Would you want to tell them about your test result, or would you rather be distracted today?

ii. What about tomorrow? Do you have work? What other plans do you have?

iii. And the day after that?

iv. When do you think you would be able to get confirmatory testing?

g. Where do you think you'll go to get confirmatory testing?

i. Do you have a good way to get there?

ii.(Explain who they can speak to at the clinic and on the phone. If it is walk-in or if an appointment is needed, if there is parking/is it reachable by public transit, etc.- - the steps needed to get tested)

h. Any other questions?

i. Ok, so I'm going to send over this information to your email address. Is it still the one I have on file? Can you give it to me to confirm?

$\mathrm{j}$. We will follow up with you in a few weeks. In the meantime, please reach out to us if you have any questions. Thank you so much for taking the time to talk today. 
Table 1 (continued)

\section{Suicide and Harm Risk Management}

1. If the participant indicates that harm to self or harm to others is a possibility. Must be extremely clear.

2. Relevant laws

a. Welfare and Institutions Code 5150

i. If as a result of a mental health condition, a person is a danger to themselves or others they may be taken into custody and placed in a facility for 72-h of treatment and evaluation.

b. Civil Code 43.92

i. If a person has communicated a serious threat of physical violence against a reasonably identifiable victim, then we should make a reasonable effort to communicate the threat to the intended victim and to the police.

3. Script:

a. It sounds like you're really upset. When you say that you want to do X, what do you mean by that?

b. Is this something that you say sometimes when you're really upset, or do you really mean you want to (Kill yourself, hurt yourself, etc., use their language).

c. [If they say "no, i'm just upset"]

i. I can understand why you'd be feeling upset. These test results are difficult to hear.

ii. How are you feeling now?

iii. How do you usually handle this feeling?

1. Do you like to be alone? Do you like to reach out to people you know? Go for a run? Something else?

2. Who do you think that you could call at a moment when you feel like this, who would be a good and supportive person to talk to? A friend or family member? A therapist or social worker? A doctor or nurse?

iv. Would you like to make an appointment with a doctor?

d. [If they express intent]

i. Have you ever tried to hurt yourself before?

ii. OK, well, it sounds like you're really upset. You've made some pretty serious statements about wanting to hurt yourself. It's definitely true that, for some people, when they are really upset, they think about [use their words]. It's my job to make sure that you don't do that. My goal is to help you stay healthy and safe, and to figure out the best way for you to cope with this news, even though you're upset. If you're serious about wanting to [use their words], I am sorry to say that I will have to break our research confidentiality and reach out to someone who can offer you more support, and especially support in person, since I'm just here on the phone. So that would be me either calling 911 and having them send an ambulance for you, or you getting yourself to a hospital and walking-in to the ER. Which would you prefer to do?

iii. [They will probably walk back the seriousness of the statement. This provides an opportunity to steer the conversation back to discussing their crisis management strategies]

iv. How can I help you get connected to someone who can give you support today?

v. (Walk through step-by-step how they can get someone next to them physically, at a minimum)

vi. (Most likely it will be hearing grief and anxiety around stigma)

\section{Reporting}

A file setup in a secure data storage system will contain the clinical notes to record the events of providing HIV test results. Plan for follow up will be included.

Enlisting emergency services warrants reporting to an IRB.

which persons can live a normal and healthy life with treatment. Participants were encouraged to reach out to friends and family members who could provide support, as well as to anyone they know who is HIV-positive who might be able to provide instrumental support through information sharing. The protocol is written in such a way as to maintain flexibility for meeting the unique needs of each individual participant and attempts to engage participants in discussion through a series of questions, allowing staff members to assess the participants' emotional response to the news, as well as their safety.

Among participants with an HIV-positive test result, 68\% ( $n=132 / 195)$ were successfully delivered to participants. Participants who did not receive their results were contacted multiple times by study staff via phone and email. Voicemails and emails from staff indicated our desire to speak with them but did not specify the reason or reference their test results. All participants who were successfully delivered a test result by phone were eligible to participate in the qualitative interview.

In total, 132 participants were invited to participate in the qualitative interview by email, approximately 3 months after receipt of their preliminary positive test results. Participants were invited in waves, and those who expressed interest were enrolled in the study. MS, JLR and CG conducted one-on-one, semi-structured, audio-recorded phone interviews between March 2018 and January 2019. During the period of interview completion, staff interviewers took notes and met regularly to compare themes arising to determine saturation. Once the interviewers felt confident that data saturation had been achieved, we ceased recruiting additional participants to interview. In total, 52 participants completed a qualitative interview; however, two interview recordings were lost due to audio file corruption, leaving an analytic sample of 50. Participants provided informed consent and were given a $\$ 40$ incentive for their 
time (approximately $1 \mathrm{~h}$ ). All procedures were approved by the IRB of the City University of New York.

\section{Measures}

Interviews were conducted via phone. Recordings were transcribed verbatim and transcripts were verified against audio recordings to ensure accuracy. Interviews followed a semistructured interview guide, which required interviewers to cover ten questions and allowed for individualized probing based on initial responses. The qualitative interview investigated missed prevention opportunities, connection to HIV care, as well as prior PrEP and PEP use and knowledge. Participants were also asked to provide feedback about their experience receiving a preliminary HIV-positive result by phone, along with any concerns they might have about that process. In this paper, we present data from these latter two questions.

Demographics and behavioral characteristics (see Table 2) were derived from data collected (via online survey) during enrollment.

\section{Data Analysis}

An inductive, thematic approach was used to analyze participants' reflections about receiving an HIV-positive result by phone $[41,42]$. ABD performed an initial close read of 50\% of the transcripts, during which, initial codes were identified inductively and categorized thematically [42]. Preliminary codes were then applied to the entire dataset. All code applications were then reviewed by CAM. Several peer debriefing sessions between ABD and CAM led to minor adjustments in our codebook and the inclusion of additional inductively identified codes that emerged through several readings of the transcripts. Peer debriefing discussions between ABD and CAM eventually resulted in $100 \%$ agreement of final code applications. The final codebook consisted of 15 codes, which were organized in four main themes: (1) acceptability of phone delivery, (2) strengths, (3) concerns, and (4) suggestions.

In addition, as another method of evaluating acceptability, an outlined criterion was used to categorize participant responses along an ordinal ranking system. Across all of their responses to relevant questions, participants' views were coded as either "preferring" phone delivery of HIV positive test results, finding the method "acceptable," or "not preferring" it. Participants were not asked directly about their preferences. For a participant to be categorized as "preferring" a phone delivery, they had to explicitly state a preference for phone-delivery in exchange of other delivery methods (e.g., in a clinic, by a doctor, via portal). For a participant's response to indicate an "acceptable" ranking, they had to express that their evaluation of this mode of
Table 2 Demographic characteristics of participants, $N=50$

\begin{tabular}{|c|c|}
\hline Characteristics & $\mathrm{M} \pm \mathrm{SD}$ or $n(\%)$ \\
\hline Age & $31.70 \pm 8.06$ \\
\hline \multicolumn{2}{|l|}{ Race/ethnicity } \\
\hline Black & $8(16 \%)$ \\
\hline Latino & $12(24 \%)$ \\
\hline White & $24(48 \%)$ \\
\hline Asian pacific islander & $6(12 \%)$ \\
\hline \multicolumn{2}{|l|}{ Gender } \\
\hline Cisgender male & $50(100 \%)$ \\
\hline \multicolumn{2}{|l|}{ Sexual orientation } \\
\hline Gay, queer & $47(94 \%)$ \\
\hline Bisexual & $3(6 \%)$ \\
\hline \multicolumn{2}{|l|}{ Education } \\
\hline High School diploma, GED or less & $7(14 \%)$ \\
\hline Some college & $29(58 \%)$ \\
\hline 4-year college degree & $11(22 \%)$ \\
\hline Graduate school & $3(6 \%)$ \\
\hline \multicolumn{2}{|l|}{ Income } \\
\hline Less than $\$ 10,000$ & $10(20 \%)$ \\
\hline$\$ 10,000-\$ 19,999$ & $12(24 \%)$ \\
\hline$\$ 20,000-\$ 29,999$ & $9(18 \%)$ \\
\hline$\$ 30,000-\$ 39,999$ & $8(16 \%)$ \\
\hline$\$ 40,000-\$ 49,999$ & $3(6 \%)$ \\
\hline$\$ 50,000-\$ 74,999$ & $6(12 \%)$ \\
\hline$\$ 75,000$ or more & $2(4 \%)$ \\
\hline \multicolumn{2}{|l|}{ Last HIV test } \\
\hline $1-3$ months ago & $3(6 \%)$ \\
\hline $3-6$ months ago & $6(12 \%)$ \\
\hline $7-12$ months ago & $8(16 \%)$ \\
\hline 1 to 2 years ago & $15(30 \%)$ \\
\hline More than 2 years ago & $11(22 \%)$ \\
\hline I've never been tested & $7(14 \%)$ \\
\hline \multicolumn{2}{|l|}{ Health insurance } \\
\hline Yes & $27(54 \%)$ \\
\hline No & $21(42 \%)$ \\
\hline I don't know & $2(4 \%)$ \\
\hline \multicolumn{2}{|l|}{ Primary care provider } \\
\hline Yes & $26(52 \%)$ \\
\hline No & $24(48 \%)$ \\
\hline
\end{tabular}

results delivery was neutral to positive, but not indicate a specific preference for it. For a participant's response to be categorized as "not preferred," they had to express a preference for another delivery method or recount a negative experience with the mode of delivery. These categories were established inductively, as most participants seemed to instinctively compare phone delivery to other potential delivery methods. Of note, four participants were not ranked ordinally due to insufficient data. 
In an attempt to address the etic positionality of the researchers, $A B D$ and $C A M$ paid critical attention to their study team identities, specifically during the code application process [43]. This is particularly pertinent, as code applications, in some cases, evaluate the effectiveness of close colleagues delivering preliminary HIV-positive results. Thus, presenting a natural conflict. Reflexive conversations occurred during peer debriefing sessions, which focused on challenging the application of our ordinal ranking criterion in the interest of analytic trustworthiness. Further, neither the primary nor secondary coder were directly involved in the delivery of HIV-positive results.

\section{Results}

The mean age of participants was 31.7 years $(\mathrm{SD}=8.0)$, and $52 \%$ of participants were men of color, with all participants identifying as cis-gender male. In total, $48 \%$ of participants were White $(n=24), 16 \%$ were Black $(n=8), 24 \%$ were Latino $(n=12)$ and $12 \%$ were Asian/Pacific Islander $(n=6)$. Most participants (94\% or $n=47$ ) identified as gay, and $6 \%$ identified as bisexual $(n=3)$. Proximate estimates of healthcare access revealed that $42 \%$ of participants were uninsured and $48 \%$ did not have a primary care doctor. Further, $34 \%$ of participants reported testing for HIV within the year prior to enrollment; $52 \%$ reported testing more than a year ago; and 14\% said they had never tested for HIV. See Table 2 for additional demographics.

\section{Acceptability of Phone Delivery}

Ordinal rankings revealed that $39 \%(n=18 / 46)$ expressed that they thought that receiving results by phone was "preferred" to other delivery methods. Similarly, $45 \%$ of participants identified delivery of preliminary positive results as "acceptable" by phone $(n=21 / 46)$. However, $15 \%(n=7 / 46)$ expressed that they would have preferred to receive their preliminary results through other delivery methods (e.g., in-person, through a portal, etc.), or recounted a negative experience receiving their results by phone, and thus were included in this group.

"I kind of prefer things from a distance, I guess, if you will, instead of being there in person... I would've felt like more 'on the spot' or ... more nervous..." (Age 24, White).

"I didn't have any opinion either way... I don't think that's an issue, delivering the results over the phone. I mean, it worked for me." (Age 31, White).

"Over the phone seems not as great as face-to-face. Just having that face-to-face support." (Age 27, White).

\section{Strengths of Phone Results Delivery}

Participants identified several strengths of our phone delivery protocol. Many participants reported that the remote delivery method allowed them time, space, and comfort to process the news of their potential diagnosis.

"I do like that a whole lot more, just to be in the comfort of my home and it's either a phone call or I call in to get results." (Age 28, Black).

"I think it was better. It gave me like a few minutes to like fall apart before I went and talked to somebody else." (Age 22, Multiracial/Other).

Additionally, participants frequently expressed that the project staff member was supportive, caring and helpful during the process of delivering their preliminary result. In these cases, some participants explained that they felt the staff member genuinely cared about them and expressed appropriate concern and sensitivity during the process.

"She talked to me like I was an actual human being and it wasn't just, 'Oh, here's your results. Bye.' She was actually able to counsel me. She asked how I felt about the news and she was actually very, very great when doing it." (Age 30, White).

Participants also expressed that they valued the information they received during the delivery call, including general information about HIV and referrals to confirmatory testing and care.

"No, I mean, it was actually really good, she was really good at explaining stuff and providing contact information and following-up and everything." (Age 27, Black).

Although not as frequently endorsed, some participants reported that they appreciated the convenience of receiving results by phone, noting that scheduling and attending in-person medical visits can be difficult. Of note, it appeared that these participants were generally referring to the entire process of testing remotely, and not just specifically receiving their HIV-positive test results by phone.

"That actually went very well. I think it was easier to do the testing at home and send it out than it would have been to make myself go into a clinic." (Age 26, White).

Further, several participants expressed that they believed this method of results delivery was more confidential then other modes (including by email or letter).

"I think it's safer than having you send me an e-mail.

Because if you send me an email, 'Hey dude! You're HIV-positive,' you know, then my older brother can 
grab my phone and see my e-mail, or whatever." (Age 29, Latino).

\section{Concerns}

Participants also reported several concerns about the process of delivering HIV results by phone. The most frequently cited concern was about the logistics of receiving a phone call during the day when they were at work or some other non-private location. Specifically, participants expressed concern that frequent screening of calls and missed calls could act as barriers to receiving results by phone. Additionally, some participants explained that they received their results at an inopportune time of day (i.e. early in the morning or during work).

"Well, my issue was, if I don't know the phone number [on the caller ID], I don't answer my phone. And for a while there they were calling, but because I did not know that number..." (Age 35, White).

A few participants expressed concerns regarding the confidentiality of delivering HIV-positive results by phone, including the need to confirm the identity of the individual being delivered results and concerns about whether the call was being recorded.

"I mean I guess I could say... what if my boyfriend had answered the phone and pretended to be me and he could have breached confidentiality that way." (Age 43, Other/Multiracial).

Further, some participants expressed concerns for the safety of those receiving HIV-positive result by phone. In some cases, they expressed that it worked fine for them, but noted that other people receiving similar results may require in-person support to ensure their safety.

"I mean you don't have that initial relationship with the person so you don't know how they're going to react to it, so you tell them something that they're not going to like and how they're going to react to it... whether it be that they may hurt themselves."(Age 26, White).

Additionally, several participants expressed that they felt awkward or unprepared to have conversations over the phone, which made this avenue of delivery challenging for them.

"It's kind of hard to talk on the phone with somebody that I don't know... "(Age 25, Latino,)

\section{Suggestions}

Participants offered suggestions to improve phone delivery of HIV-positive test results. Several participants suggested that greater follow-up call frequency after the delivery of their result would have improved their experience. Further, some participants emphasized the importance of offering support during the call, suggesting that greater efforts in this area would be beneficial. Additionally, one participant highlighted the importance of reiterating that HIV is now a treatable, chronic disease, saying:

"I have a long-time friend that has HIV and he heard over the phone. And it didn't go as good as my reaction [my experience]... [Unlike him, I] know a little about it [HIV] and how it's manageable."(Age 27, White).

Additionally, a few participants expressed a desire to receive their test results via email or an online portal, instead of speaking on the phone with a staff member. Participants suggesting such options were, in some cases, also those who reported discomfort speaking by phone. However, these sentiments appeared to be overshadowed by many participants stressing the importance of emotional support during the results delivery, including having someone to listen and respond to their concerns, which would not be possible to deliver through an online portal.

"I guess you all could've done maybe like email, some type of secure email, since I always check email. Maybe somewhere to $\log$ in and not have to physically tell it over the phone." (Age 29, Black).

\section{Discussion}

Expanding HIV testing is critical to stemming the spread of HIV in vulnerable populations, such as MSM. One method of expanding HIV testing would be to increase remote testing opportunities, such as through the use of mail-in testing and phone delivery of HIV-positive results. Phone delivery of HIV-positive results may remove some obstacles to testing, while preserving core elements of in-person HIV counseling like social support and direct referral to care. In the past, public health authorities have been reluctant to roll-out such strategies. However, in the current digital age and especially given the COVID-19 pandemic [44], this may be an opportune moment for the expansion of telehealth into HIV testing both for healthcare and research.

Participants by-and-large deemed phone delivery of HIV-positive results to be acceptable. The men highlighted the quality of the delivery of their preliminary test results, emphasizing that their results were delivered with care and concern. Nevertheless, some participants expressed 
concerns, particularly with regard to confidentiality and conveyed that face-to-face delivery might offer greater emotional support than can be had over the phone. In response to these shortfalls, several participants expressed that phonedelivery was not a good avenue to receive a positive test result. Although our results offer preliminary support for remote HIV-positive results delivery, further research is required to assess the acceptability of this mode of results delivery across diverse populations vulnerable to HIV.

Participants identified several strengths unique to delivering HIV-positive test results by phone, including that receiving results outside of a clinical setting afforded comfort, surmounted obstacles to in-person testing and results delivery, and maintained confidentiality. Participants reported that returning in-person to receive HIV test results was often challenging and inconvenient; highlighting that phonedelivery may overcome barriers to receiving test results via traditional clinical settings. Indeed, prior research reveals many logistical barriers to attending clinical appointments for HIV testing, as well as results delivery [13, 14, 20]. However, opinions about confidentiality were more nuanced, with only some participants believing that confidentiality improved through phone delivery of results, and others expressing concerns about maintaining confidentiality using this approach. Studies report that confidentiality is especially important for young MSM, who may be less likely to seek testing when services are not perceived as confidential $[45,46]$. This is particularly relevant, as the current HIV epidemic disproportionately impacts young MSM [1]. Thus maintaining confidence in confidentiality may be key to remote testing with young people vulnerable to HIV.

Other concerns unique to phone delivery were related to answering phone calls, the level of support afforded via phone conversations, and potential safety challenges resulting from remote delivery. Concerns included logistical issues with receiving a test result call at an inopportune time of day, as well as receiving calls from an unknown number and speaking to a stranger over the phone. With increasing use of text message, email, and other messaging apps, phone calls have become increasingly outmoded in the age of smartphones, with Americans answering fewer than half of incoming phone calls [47]. Contemporary smartphone use could prove problematic for delivering HIV test results over the phone to a generation of cell phone users who screen calls, particularly from unfamiliar phone numbers. Although a randomized controlled trial with homeless youth conducted in 2002 revealed a higher rate of results delivery by phone versus in-person, further study of phone delivery in the age of smartphones is warranted.

Additionally, some participants expressed concerns about potential safety issues resulting from remote delivery of HIV-positive results. However, in our study, as of August 2020 , we have yet to enlist emergency services with our racially and ethnically diverse sample of participants from across the U.S. With the emergence of rapid home-testing, concerns arose among public health professionals and HIV counselors, specifically around suicide risk [48]. Concerns about heightened risk of suicidality as a result of receiving a positive result outside of a clinical setting have not been substantiated by data [30]. However, a 2017 study of MSM in Buenos Aires revealed that, of 500 respondents, 9\% estimated that they would attempt suicide in the hypothetical event of receiving a positive, rapid HIV test result while alone. This finding supports that some interpersonal connection may be protective for a subset of vulnerable MSM testing positive for HIV, and that more research is needed to determine the amount and nature of contact that is helpful to MSM receiving HIV-positive results via phone [49].

In addition to perceived benefits of phone delivery, participants reported several strengths to the process that were specific to our protocol. This included support given by staff members, information about HIV, and referral to confirmatory testing and care. Further, one participant highlighted that phone-delivery of HIV-positive test results was acceptable in light of developments in HIV care that make the virus a manageable condition. Advances in HIV treatment mean that patients diagnosed with HIV can live long, healthy lives with adherence to antiretroviral therapy (ART). In the age of ART, an HIV diagnosis is more akin to other chronic diseases that require ongoing medication management to achieve optimal clinical outcomes. Thus, the delivery of HIV-positive test results should be considered within a contemporary context, while remaining cognizant of the social stigma that still accompanies the diagnosis in many communities [48]. Our protocol highlights the manageable nature of HIV, while emphasizing the importance of confirmatory testing and connection to care, as well as encouraging participants to reach out to friends and family who can provide safe support. These components of HIV positive test result delivery may be integral to participants receiving the news in a manner that they experience as supportive and responsive.

Participants also expressed concerns that reflected shortcomings of our protocol. Several participants underscored that greater emotional support during delivery and more frequent follow-up calls, would have improved their experience. Indeed, prior research supports that medical provider tone and approach during the delivery of HIV positive test results can affect patient experience [50, 51]. For example, results from a qualitative study found that aggravated or upset providers negatively affected the experience for patients receiving their HIV test results [50]. Although our protocol was built with the intention of providing strong interpersonal support via phone, some participants may have greater support needs than others. Thus, our delivery protocol should be used with the understanding that adjustments may be made based on individual needs and contexts. 


\section{Limitations}

Our results should be considered in light of their limitations. First, recruitment ads emphasized "free at-home HIV testing" as well as compensation for completing study assessments. Those with the most severe HIV testing anxiety may not have enrolled in our cohort from the outset and so those perspectives may be less likely to be represented in our sample. That said, staff communication with participants reveals that many participants with HIV-related anxiety are, in fact, enrolled in the cohort. Second, only those who were successfully delivered their HIV test result by phone were asked to participate in this interview. Thus, invited participants are likely different from the larger subsample of participants who received a preliminarily positive HIV test result. For example, we contacted participants for whom we received a preliminary positive test result many times in an attempt to deliver their results. Those who did not respond to our outreach may have other concerns about the remote results delivery process that are not fully represented here. Additionally, participants who agreed to complete our qualitative interview were likely more willing to discuss their experience receiving their results and, thus, may not reflect the larger group of participants who were delivered HIV positive results by phone. These participants may have had a better experience receiving their results by phone, making it more likely that they would participate in the interview.

Further, social desirability bias may also be operating, as study staff were asking participants to evaluate the performance of other study staff and study procedures. Although the staff who delivered results did not conduct interviews with participants to whom they delivered results, it is possible that participants did not feel comfortable providing feedback critical of the study directly to staff members. However, our interview guide only probed for concerns regarding phone delivery of HIV positive test results and, overall, participants seemed largely willing to critique the process and offer suggestions. Last, the data in this study are primarily qualitative and thus useful for theory and hypothesis generation. That said, further quantitative research is needed, and may benefit from using our findings to develop measures that assess the acceptability and feasibility, including strengths and weaknesses, of phone delivering HIV-positive test results.

\section{Conclusion}

Our findings reveal that phone delivery of preliminary HIVpositive test results is generally acceptable. As strengths of this method of results delivery, participants cited compassionate delivery of their results, and staff provision of important information about HIV and treatment engagement, as well as the ability to emotionally process the information in the privacy of their own space. Nevertheless, some expressed logistical concerns about receiving the phone call and safety concerns, as unique issues with this mode of test result delivery. Current HIV testing options include testing in-person at a clinic or provider's office, as well as rapid at-home testing and mail-in testing services. The emergence of an array of testing options call for greater understanding of the acceptability of novel avenues of HIV results delivery. Phone delivery of HIV-positive results may obviate some of the hurdles to in-person testing, while maintaining direct linkage to care and some of the features of emotional support afforded by in-person counseling.

Acknowledgements Together 5000 was funded by the National Institutes for Health (UH3 AI 133675-PI Grov). Other forms of support include the CUNY Institute for Implementation Science in Population Health and the Einstein, Rockefeller, CUNY Center for AIDS Research (ERC CFAR, P30 AI124414). Special thanks to additional members of the T5K study team: Gloria Perez, Sarit A. Golub, Gregorio Millett, Don Hoover, Sarah Kulkarni, Chloe Mirzayi, and Pedro B. Carneiro. Thank you to the members of our Scientific Advisory Board: Adam Carrico, Michael Camacho, Demetre C. Daskalakis, Sabina Hirshfield, Jeremiah Johnson, Claude Mellins, and Milo Santos. We are grateful also for help from program staff at NIH: Gerald Sharp, Sonia Lee, and Michael Stirratt. Although the NIH financially supported this research, the content is the responsibility of the authors and does not necessarily reflect official views of the NIH.

\section{References}

1. Centers for Disease Control and Prevention. HIV Surveillance Report, 2017. 2018; https://www.cdc.gov/hiv/pdf/library/repor ts/surveillance/cdc-hiv-surveillance-report-2017-vol-29.pdf. Accessed 25 March 2020.

2. The Williams Institute. How Many People are Lesbian, Gay, Bisexual, and Transgender? 2011; https://williamsinstitute.law. ucla.edu/publications/how-many-people-lgbt/. Accessed 20 May 2020.

3. DiNenno EA, Prejean J, Irwin K, et al. Recommendations for HIV screening of gay, bisexual, and other men who have sex with men-United States, 2017. MMWR Morb Mortal Wkly Rep. 2017;66(31):830.

4. Nelson KM, Pantalone DW, Gamarel KE, Carey MP, Simoni JM. Correlates of never testing for hiv among sexually active internetrecruited gay, bisexual, and other men who have sex with men in the United States. AIDS Patient Care STDs. 2018;32(1):9-15.

5. Noble M, Jones AM, Bowles K, DiNenno EA, Tregear SJ. HIV testing among internet-using MSM in the United States: systematic review. AIDS Behav. 2017;21(2):561-75.

6. Clark HA, Oraka E, DiNenno EA, et al. Men who have sex with men (MSM) who have not previously tested for HIV: results from the MSM testing initiative, United States (2012-2015). AIDS Behav. 2019;23(2):359-65.

7. Sanchez TH, Sineath RC, Kahle EM, Tregear SJ, Sullivan PS. The annual American men's internet survey of behaviors of men who have sex with men in the United States: protocol and key indicators report 2013. JMIR Publ Health Surveill. 2015;1(1):e3.

8. Group ISS. Initiation of antiretroviral therapy in early asymptomatic HIV infection. New England J Med. 2015;373(9):795-807. 
9. Marks G, Crepaz N, Janssen RS. Estimating sexual transmission of HIV from persons aware and unaware that they are infected with the virus in the USA. AIDS. 2006;20(10):1447-500.

10. Colfax GN, Buchbinder SP, Cornelisse PG, Vittinghoff E, Mayer $\mathrm{K}$, Celum C. Sexual risk behaviors and implications for secondary HIV transmission during and after HIV seroconversion. AIDS. 2002;16(11):1529-35.

11. Higginbotham $\mathrm{S}$, Holmes $\mathrm{R}$, Stone $\mathrm{H}$, et al. Adoption of protective behaviors among persons with recent HIV infection and diagnosisAlabama, New Jersey, and Tennessee, 1997-1998. MMWR. Morb Mort Wkly Rep. 2000;49(23):512-5.

12. Weinhardt LS, Carey MP, Johnson BT, Bickham NL. Effects of HIV counseling and testing on sexual risk behavior: a meta-analytic review of published research, 1985-1997. Am J Publ Health. 1999;89(9):1397-405.

13. Philbin MM, Tanner AE, DuVal A, et al. Factors affecting linkage to care and engagement in care for newly diagnosed HIVpositive adolescents within fifteen adolescent medicine clinics in the United States. AIDS Behav. 2014;18(8):1501-10.

14. Brimlow DL, Cook JS, Seaton R. Stigma, HIV/AIDS: A review of the literature. US Department of Health Human Services. 2003.

15. Kellerman SE, Lehman JS, Lansky A, et al. HIV testing within at-risk populations in the United States and the reasons for seeking or avoiding HIV testing. J Acquir Immune Defic Syndr. 2002;31(2):202-10.

16. Horridge DN, Oh TS, Alonzo J, et al. Barriers to HIV testing within a sample of spanish-speaking latinx gay, bisexual, and other men who have sex with men: implications for hiv prevention and care. Health Behav Res. 2019. https://doi. org/10.4148/2572-1836.1069.

17. Burnett JC, Broz D, Spiller MW, Wejnert C, Paz-Bailey G. HIV Infection and HIV-associated behaviors among persons who inject drugs - 20 cities, United States, 2015. MMWR Morb Mortal Wkly Rep. 2018;67(1):23-8.

18. Pharr JR, Lough NL, Ezeanolue EE. Barriers to HIV testing among young men who have sex with men (MSM): experiences from clark county. Nevada Glob J Health Sci. 2015;8(7):9-17.

19. Levy ME, Wilton L, Phillips G 2nd, et al. Understanding structural barriers to accessing HIV testing and prevention services among black men who have sex with men (BMSM) in the United States. AIDS Behav. 2014;18(5):972-96.

20. Schafer KR, Albrecht H, Dillingham R, et al. The continuum of HIV care in rural communities in the United States and Canada: what is known and future research directions. J Acquir Immune Defic Syndr. 2017;75(1):35.

21. Guidelines for Using HIV Testing Technologies in Surveillance: Selection, Evaluation and Implementation: 2009 Update. Current HIV Testing Technologies and Strategies Used in Surveillance 2009; https://www.ncbi.nlm.nih.gov/books/NBK305269/.

22. Franco-Paredes C, Tellez I, del Rio C. Rapid HIV testing: a review of the literature and implications for the clinician. Curr HIV/AIDS Rep. 2006;3(4):169-75.

23. Golub SA, Gamarel KE. The impact of anticipated HIV stigma on delays in HIV testing behaviors: findings from a community-based sample of men who have sex with men and transgender women in New York City. AIDS Patient Care STDs. 2013;27(11):621-7.

24. CDC. Self-Testing. HIV 2020; https://www.cdc.gov/hiv/testing/ self-testing.html. Accessed 25 March 2020.

25. HIV Test Kit. 2020; https://www.mylabbox.com/product/ hiv-home-test-kit/.

26. Walensky RP, Paltiel AD. Rapid HIV testing at home: does it solve a problem or create one? Ann Intern Med. 2006;145(6):459-62.

27. Lippman SA, Périssé ARS, Veloso VG, et al. Acceptability of self-conducted home-based HIV testing among men who have sex with men in Brazil: data from an on-line survey. Cad Saude Publica. 2014;30(4):724-34.

28. Choko AT, Desmond N, Webb EL, et al. The uptake and accuracy of oral kits for HIV self-testing in high HIV prevalence setting: a cross-sectional feasibility study in Blantyre, Malawi. PLoS Med. 2011;8(10):e1001102-e10011021001102.

29. The Centers for Disease Control and Prevention. Revised Guidelines for HIV Counseling, Testing, and Referral. 1999; https:// www.cdc.gov/mmwr/preview/mmwrhtml/rr5019a1.htm.

30. Myers JE, El-Sadr WM, Zerbe A, Branson BM. Rapid HIV self-testing: long in coming but opportunities beckon. AIDS. 2013;27(11):1687-95.

31. Grov C, Westmoreland D, Rendina HJ, Nash D. Seeing Is Believing? unique capabilities of internet-only studies as a tool for implementation research on HIV prevention for men who have sex with men: a review of studies and methodological considerations. JAIDS J Acquir Immune Defic Syndr. 2019;82:S253-S260260.

32. NIH. Limited Interaction Targeted Epidemiology (LITE) to Advance HIV Prevention (UG3/UH3). 2016; https://grants.nih. gov/grants/guide/rfa-files/rfa-ai-16-031.html. Accessed 25 March 2020.

33. Nash D, Stief M, MacCrate C, et al. A web-based study of HIV prevention in the era of pre-exposure prophylaxis among vulnerable HIV-negative gay and bisexual men, transmen, and transwomen who have sex with men: protocol for an observational cohort study. JMIR Res Protoc. 2019;8(9):e13715.

34. LITE GRANT: Large-scale study of HIV-negative MSM across the nation. 2020; https://www.prideresearch.org/lite-grant.html.

35. Tsu RC, Burm ML, Gilhooly JA, Sells CW. Telephone vs faceto-face notification of HIV results in high-risk youth. J Adolesc Health. 2002;30(3):154-60.

36. McKinstry LA, Goldbaum GM, Meischke HW. Telephone notification of HIV test results: impact in King County Washington. Sexual Transm Dis. 2007;34(10):796-800.

37. Martin L, Knight V, Read PJ, McNulty A. Clients' preferred methods of obtaining sexually transmissible infection or HIV results from Sydney Sexual Health Centre. Sexual Health. 2013;10(1):91-2.

38. Grov C, Westmoreland DA, Carneiro PB, et al. Recruiting vulnerable populations to participate in HIV prevention research: findings from the Together 5000 cohort study. Annals of epidemiology. 2019.

39. Grov C, Stief M, Westmoreland DA, MacCrate C, Mirzayi C, Nash D. Maximizing response rates to ads for free at-home HIV testing on a men-for-men geosocial sexual networking app: lessons learned and implications for researchers and providers. Health Educ Behav. 2020;47(1):5-13.

40. Posner K, Brown GK, Stanley B, et al. The columbia-suicide severity rating scale: initial validity and internal consistency findings from three multisite studies with adolescents and adults. Am J Psychiat. 2011;168(12):1266-77.

41. Strauss A, Corbin J. Qualitative research: London: Sage; 1990.

42. Ryan GW, Bernard HR. Techniques to identify themes. Field Methods. 2003;15(1):85-109.

43. Watt D. On becoming a qualitative researcher: the value of reflexivity. Qual Rep. 2007;12(1):82-101.

44. Shiau S, Krause KD, Valera P, Swaminathan S, Halkitis PN. The burden of COVID-19 in people living with HIV: a syndemic perspective. AIDS Behav. 2020. https://doi.org/10.1007/s10461-02002871-9.

45. Phillips G II, Ybarra ML, Prescott TL, Parsons JT, Mustanski B. Low rates of human immunodeficiency virus testing among adolescent gay, bisexual, and queer men. J Adolesc Health. 2015;57(4):407-12. 
46. Witzel TC, Rodger AJ, Burns FM, Rhodes T, Weatherburn P. HIV self-testing among men who have sex with men (MSM) in the UK: a qualitative study of barriers and facilitators, intervention preferences and perceived impacts. PLoS ONE. 2016;11(9):e0162713-e01627130162713.

47. Hiya. State of the Phone Call. Half-Yearly Report 2019; https ://assets.hiya.com/public/pdf/HiyaStateOfTheCall2019H1.pdf. Accessed 25 March 2020.

48. Arnold C. At-home HIV test poses dilemmas and opportunities. Lancet. 2012;380(9847):1045-6.

49. Pando MA, Dolezal C, Marone RO, et al. High acceptability of rapid HIV self-testing among a diverse sample of MSM from Buenos Aires, Argentina. PloS One. 2017;12(7):e0180361-e01803610180361.
50. Hult JR, Maurer SA, Moskowitz JT. "I'm sorry, you're positive": a qualitative study of individual experiences of testing positive for HIV. AIDS Care. 2009;21(2):185-8.

51. Worthington C, Jackson R, Mill J, Prentice T, Myers T. Sommerfeldt SJAc HIV testing experiences of Aboriginal youth in Canada: service implications. AIDS Care. 2010;22(10):1269-76.

Publisher's Note Springer Nature remains neutral with regard to jurisdictional claims in published maps and institutional affiliations. 